\title{
LA PEDAGOGÍA CRÍTICA Y LAS CIENCIAS SOCIALES
}

\author{
CRITICAL PEDAGOGY AND SOCIAL SCIENCES \\ DOI: https://doi.org/10.53595/rlo.v1.i2.018
}

\author{
Dora Verónica Pinto Mamani \\ Escuela de Posgrado \\ Universidad Nacional del Altiplano \\ https://orcid.org/0000-0001-5994-9753 \\ doraveronicapintomamani05@gmail.com
}

\section{RESUMEN}

A través del presente ensayo se desarrollará un abordaje explicativo sobre la pedagogía crítica y el respaldo de los principios básicos de la teoría crítica y la tradición crítica de la educación y básicamente en el área de ciencias sociales, puesto que en la actualidad es un aspecto muy importante de discusión, ya que a pesar de que se está trabajando en el logro de las competencias, existe algunas actividades tradicionales que aun los docentes proponen a los estudiantes, en el que el profesor incluso da a conocer su postura y el educando en su gran mayoría acepta tal cual es presentado y no va más allá que simplemente un conocimiento impartido. Entonces el presente ensayo nos deja un gran desafío reflexivo de reconocer a la pedagogía crítica como parte muy importante de la teoría crítica cuyo objetivo es lograr la emancipación a través de la autonomía y la forma en que la persona asume un conocimiento cotidiano, pese a las diferencias sociales.

Palabras clave: Autodeterminación, crítico, conocimiento, emancipación, pedagogía crítica.

\begin{abstract}
Through this essay, an explanatory approach will be developed on critical pedagogy and the support of the basic principles of critical theory and the critical tradition of education and basically in the area of social sciences, since at present it is a very important aspect. important for discussion, since despite the fact that work is being done on the achievement of competences, there are some traditional activities that the teacher still proposes to the student in which the teacher even makes his position known and the student mostly accepts as it is presented and does not go beyond simply imparted knowledge.

So this essay leaves us with a great reflective challenge of recognizing critical pedagogy as a very important part of critical theory whose objective is to achieve emancipation through autonomy and the way in which the person assumes daily knowledge, despite the social differences.
\end{abstract}




\section{INTRODUCCION}

Ahora que a nivel de la educación se habla de direccionar nuestras experiencias de aprendizaje a los enfoques transversales, tal es el caso del enfoque intercultural que está orientado a una convivencia basada en el acuerdo y la complementariedad, así como en el respeto a la propia identidad y a las diferencias. Es oportuno examinar el objetivo de la pedagogía crítica para el desarrollo de ciudadanos consientes respecto a un conocimiento que imparte un maestro en las escuelas, referente a las distintas posturas que ofrece, y las que el estudiante podría asumir desde su situación.

El presente ensayo presenta una parte teórica que aborda sobre qué es y lo que aborda la pedagogía crítica, en segundo lugar, se conocerá los principios de la teoría crítica, que busca la emancipación de la persona a través de la educación, en el tercer punto las pretensiones de las ciencias sociales basados en la pedagogía crítica, los enfoques y las competencias que permite desarrollar.

\section{DESARROLLO}

\section{La pedagogía critica}

Según Valencia (2016), la pedagogía crítica es una propuesta de enseñanza que pretende formar y transformar seres humanos con autonomía y autodeterminación y que a la vez desarrolle la capacidad de ser crítico, a lo que Magendzo (2003), refiere que la pedagogía critica está íntimamente relacionado con la teoría crítica; cuyo objetivo es crear una sociedad más justa a través de la emancipación del oprimido y empoderar a un nivel que sea capaz de transformar su situación en la que convive. Aquella emancipación que surja de la conciencia, de las acciones liberadoras y coerciones escondidas (Habermas, 1987). Por su parte Ramírez (2008), señala que la pedagógica critica también se considera como un paradigma del ejercicio del docente, cuyo punto eje del proceso de formación es para quién, por qué, cómo, cuándo y dónde se desarrolla tal ejercicio pedagógico. Así mismo Giroux (1983) afirma que la pedagogía crítica pretende crear nuevas formas de conocimiento rompiendo disciplinas e interrelacionando disciplinas y planteando preguntas referentes a los centros de poder y grupos populares, Por lo que, McLaren (1984) menciona las principales interrogantes y básicamente el cómo y el por qué dicho conocimiento es construido como lo formalmente aceptado por la cultura dominante; el cómo nuestro conocimiento cotidiano o nuestras construcciones sociales es vivido y producido en otros términos ¿cuál es el objetivo de la existencia del conocimiento popular?. 
En fin, nadie puede dudar que el enseñar y aprender es fundamental para que las sociedades vayan progresando y se vayan formando ciudadanos de acuerdo a la exigencia social, sin embargo, con la pedagogía critica se pretende llevar a la persona a pensar críticamente de los individuos y su accionar por lo tanto según Giroux (1983) es importante proporcionar una nueva forma de lectura de las diferentes áreas para lograr un aprendizaje significativo cuyo propósito es recuperar el poder y la identidad a partir del conocimiento cotidiano.

El principal objetivo de la pedagogía crítica es: transformar el sistema educativo tradicional, incentivar el cuestionamiento de lo impartido, ser aplicada de forma ética y política, fomentar en el alumnado interrogantes, cuestionamientos sobre sus acciones sociales, potenciar métodos de enseñanza desde una postura analítica, transformar los valores y las prácticas educativas y propiciar cambios sociales cuestionando los procesos políticos y sociales.

\section{Teoría crítica}

Según Frankenberg (2011), la teoría crítica de la escuela de Frankfurt se originó en la década de 1920 bajo la dirección del gran filósofo Horkheimer como crítica sistemática a la reducción del conocimiento empírico de la realidad reconociendo a la razón humana como la capacidad intelectual para el análisis de la realidad. Que según Mora (2009) pretendía lograr un mundo altamente independiente a través de un proceso igualitario que requiere una larga formación y educación crítica y emancipadora.

Según Valencia (2016), la teoría crítica integrada a la didáctica, habla de una estrecha relación entre el proceso de enseñanza y aprendizaje, predominando el vínculo desde la dialéctica entre los dos agentes educativos, tal es el caso docente - estudiante; en el cual, Gallo (2008), sostiene que el docente es el encargado de apropiarse el contenido, para reconocer el sentido que tiene, para luego orientar hacia un aprendizaje formativo, asumiendo el rol de mediador que da la oportunidad al estudiante de apropiarse de sí mismo y del conocimiento real. En términos de Comenio (1982) el docente debe lograr que el estudiante crezca a partir de su autonomía, voluntad y formación; y no se observe al estudiante como una tabla rasa libre de cuestionamientos sobre los hechos que se ha vivido, lo que se está viviendo y lo que podríamos vivir, el mismo que nos permite observar los grandes cambios de la educación basado en una formación critica. Finalmente, Munck (2010), se cuestiona si la teoría crítica puede enfrentar dichos desafíos.

\section{Principios de la teoría critica}


Lorenzo (2015), manifiesta que estos principios hacen posible que los estudiantes reflexionen y transformen la forma de su pensamiento y al respecto los docentes debemos ser grandes investigadores para comprender la situación en la que están viviendo los estudiantes, asimismo la participación y la relación entre docente y estudiante durante el proceso de enseñanza y aprendizaje en los espacios de la institución deben ser vitales para transformar valores, ya que la teórica crítica requiere de las experiencias para el logro de la transformación, obligando a que la persona sea parte de la discusión de forma libre, conduciendo su argumento a partir de dogmas religiosos, ideológicos o disciplinares sin sentirse presionado y obligado.

\section{Las ciencias sociales y la crítica}

Según Galafassi (2002), las ciencias sociales son aquellos aconteceres que se relacionan con el comportamiento de las personas, incluso el de toda una sociedad que está en una constante lucha para el logro de la misión y visión compartida, por lo tanto, podemos decir que las instituciones requieren de un aspecto muy importante, tal es el caso del modelo educativo que asume cada institución, a lo que las escuelas públicas de los países más desfavorecidos suelen limitarse al poder de leer y escribir y en casos muy extremos lograr una educación secundaria, mientras que las familias pudientes pueden llegar fácilmente a una educación superior; que según Valencia (2016), se encuadra en el saber y la forma en que recrea los contenidos de formación, dejando de lado el típico procedimiento de que el docente lo transmita y más bien lograr que el estudiante pueda apropiarse del conocimiento a través de cualquier medio y de forma voluntaria, haciendo tendencia de aquel principio de la educación que es el de aprender a aprender; para lograr entonces una educación critica es necesario el alto grado de disposición y compromiso. Este aspecto desde el punto de vista sociológico, que según Montero (1992) es la sociedad quien alcanzan su punto de crítica a través de una acción comunicativa y manifestaciones más estridentes.

Para Tamayo (2015), desde la didáctica es un factor muy importante la enseñabilidad en el proceso de enseñanza como una estrategia que hace posible reflexionar sobre el objeto de estudio, respecto a esto Habermas (1987), afirma que es muy importante educar en la autonomía para su liberación proporcionando al estudiante una comprensión precisa y profunda de la historia para así lograr los cambios personales y sociales a lo que Giroux (1983), plantea proporcionar a los estudiantes una forma de leer la historia que les permitirá recuperar el poder y la identidad, que consiste en rechazar las diferencias entre 
la cultura superior y cultura popular, haciendo que el currículo responda al conocimiento cotidiano y la ética, respetando las prácticas culturales particulares.

A toda esta actividad Avendaño \& González (2020) concluyen que la persona en un proceso de lectura debe ser inferente, analizativo, capaz de sintetizar y evaluar la lectura histórica en cuestión; para que así también sea claro y plantear su postura con una madurez emocional y ética. Hecha las investigaciones correspondientes a los diferentes autores sería necesario plantear la pedagogía crítica como modelo educativo de las instituciones, para el desarrollo de un pensamiento de libertad desde una experiencia interrelacionadas de las áreas priorizadas de la educación básica regular.

\section{CONCLUSIONES}

A partir de la exposición sobre la pedagogía crítica, se puede afirmar que, es una propuesta que facilita el proceso de enseñanza y aprendizaje en base al reconocimiento de una persona o estudiante, como un agente de cambio para la sociedad logrando solucionar los problemas desde la teoría y la práctica.

Desde el punto de vista de la teoría crítica y las Ciencias Sociales podemos concluir que las personas debemos ser capaces de descubrir, reflexionar sobre el sentido oculto de un acontecer social e histórico, asumiendo una postura crítica.

\section{REFERENCIAS}

Avendaño, G. S., \& González, O. L. (2020). Una propuesta de plan de clase para dinamizar la lectura crítica. Folios, 52, 155-171. https://doi.org/10.17227/folios.52-10002

Comenio, J. (1982). Didáctica Magna. México: Porrúa.

Frankenberg, G. (2011). Teoría crítica. Academia: Revista Sobre Enseñanza Del Derecho de Buenos Aires, 9(17), 67-84.

Galafassi, G. (2002). La teoría crítica de la Escuela de Frankfurt y la crisis de la idea de razón en la modernidad.

Gallo, L. (2008). La mirada de la didáctica desde la formación categorial. Medellín: Universidad de Antioquia.

Giroux, P. (1983). Theory and resistance in education: Apedagogy for the opposition. London: Heinemann.

Habermas, J. (1987). Teoría de la acción comunicativa, Racionalidad de la acción y racionalización social. Madrid:Taurus.

Lorenzo, C. (2015). La Teoría Crítica como fundamento epistemológico de la Bioética: una propuesta. Revista Colombiana de Bioética, 6(2), 118. https://doi.org/10.18270/rcb.v6i2.831

Magendzo, A. (2003). Pedagogia critica y educación en derechos humanos. In Revista de Pedagogía Critica (Issue 2, pp. 19-27).

McLaren, P. (1984). La vida en la escuelas. La Vida En Las Escuelas, 203-234.

Montero, F. (1992). Mundo y accion comunicativa según Habermas. Fragmentos de Filosofía, 1(1992), 149-166.

Mora, D. (2009). Pedagogía y Didáctica Crítica para una Educación Liberadora. Revista Integra Educativa, 2(1), 25-60.

Munck, R. (2010). La teoría crítica del desarrollo: resultados y prospectiva. Migración y Desarrollo, 08(14), 35-57. https://doi.org/10.35533/myd.0814.rm

Ramírez, R. (2008). La pedagogía crítica Una manera ética de generar procesos educativos. Revista Folios, 28, 108. https://doi.org/10.17227/01234870.28folios108.119

Tamayo, J. (2015). La didáctica en la pedagogía crítica, una apuesta para la permanencia. 7511. http://revistavirtual.ucn.edu.co/index.php/RevistaRyS/article/view/782/1308\%0ALa 
Valencia, M. (2016). La Didáctica en la pedagogía crítica, una apuesta para la permanencia. Revista Reflexiones $y \quad$ Saberes, 28-32. http://revistavirtual.ucn.edu.co/index.php/RevistaRyS/article/view/782/1308 